\title{
Low-frequency excitations in a molecular glass: Single-particle dynamics
}

\author{
F. J. Bermejo \\ Instituto de Estructura de la Materia, Consejo Superior de Investigaciones Científicas, Serrano 123, E-28006 Madrid, Spain
}

\author{
J. Alonso \\ Rutherford Appleton Laboratory, Chilton, Didcot, Oxon, OX11 0QX United Kingdom
}

A. Criado

Departamento de Física de la Materia Condensada, Universidad de Sevilla, P.O. Box 1065, E-41080 Sevilla, Spain

F. J. Mompeán

Rutherford Appleton Laboratory, Chilton, Didcot, Oxon, OX11 0QX United Kingdom

J. L. Martínez*

Institut Laue Langevin, 156X, F-38042 Grenoble CEDEX, France

M. García-Hernández and A. Chahid

Instituto de Estructura de la Materia, Consejo Superior de Investigaciones Científicas, Serrano 123, E-28006 Madrid, Spain

(Received 15 January 1992; revised manuscript received 10 April 1992)

\begin{abstract}
The low-frequency dynamics of a molecular glass former has been investigated by means of inelastic neutron scattering from a mostly incoherent sample as well as by computer molecular-dynamics simulation. The mode assignments have been carried out using the neutron data for the polycrystal as well as by means of the analysis of the density of states (DOS) computed from a lattice-dynamics calculation. The polycrystalline sample is then taken as the reference state and the deviations that are a known characteristic of the glassy phase are discussed in detail. In particular, the enhancement of the low-frequency part of the DOS corresponding to the glassy phase that gives rise to a welldefined low-frequency inelastic peak in the neutron $S(Q, \omega)$ dynamic structure factors is found to arise from translational modes that are dominant over the rotational contribution. Such modes are also found to give rise to a bump in the temperature dependence of the $C_{v} / T^{3}$ specific-heat curves at $2<T<10 \mathrm{~K}$.
\end{abstract}

\section{INTRODUCTION}

The thermal properties of glasses well below the thermodynamical glass transition temperature $T_{g}$ are now starting to be understood on a quantitative basis. ${ }^{1,2} \mathrm{Al}-$ though at temperatures below $1 \mathrm{~K}$ there is wide agreement that most of the observed phenomenology can be explained in terms of tunneling motion of some (yet to be specified) atoms, ${ }^{3}$ it seems clear that additional excitations are present at moderately high temperatures $(1-50 \mathrm{~K})$, which are responsible for the anomalous behavior of the temperature dependence of several macroscopic properties such as the specific heats or the thermal conductivity. ${ }^{4}$

Several attempts have been registered in order to quantitatively analyze the origin of the anomalies in the temperature dependence of thermodynamic (specific heats) and transport (thermal conductivity) properties at moderately low temperatures such as mode softening by strains, ${ }^{5}$ or phonon scattering by localized modes, ${ }^{6}$ although at present there seems to be no commonly accepted mechanism that can be considered to be as uni- versal as most of the properties of the glassy state are.

The present paper aims to shed some light on these problems by means of the study of the low-frequency dynamics of a molecular glass former (methanol $\mathrm{CH}_{3} \mathrm{OH}$ ). Although the glass formed by this material is substantially more complicated, due to the presence of the hydrogen-bond network, than the current van der Waals glasses such as those formed by complex organics or polymers (i.e., orthoterphenyl or polybutadiene), it was chosen for several reasons. First of all its basic molecular unit is relatively small so that it enables the simulation by means of computer molecular dynamics (MD) using a large enough number of particles. Moreover there exist some model potentials capable of accounting for most of the dynamical properties, such as the collective dynamics of the liquid phase as well as the crystal structurerelated properties as has been shown in previous work. ${ }^{7,8}$ From an experimental point of view the glass obtained by rapid quenching from room-temperature liquid is stable for days below $115 \mathrm{~K}$ in bulk samples, without showing noticeable crystallization and finally, the internal molecular modes are, in condensed phases, well separated from 
the low-frequency (lattice) ones. This material has been previously characterized by means of differential scanning calorimetry, where the $T_{g}$ was located at about 120 $\mathrm{K}$, as well as from Brillouin scattering of light. ${ }^{9}$

The present paper constitutes a first part and it is focused on the comparison of the low-frequency response of the glassy and crystalline phases as observed using high-resolution inelastic neutron scattering (INS) with the density of states calculated by means of MD simulations in the case of the glass and lattice-dynamics (LD) computations for its polycrystalline counterpart. The crystal will serve as a reference state and a comparison between the dynamical properties of the crystal and the low-temperature liquid has already been given. ${ }^{7}$ A second part containing experimental and simulation data regarding the collective response will follow in due course.

The outline of the paper is as follows: Sec. II contains some information regarding the experimental, data treatment, and computational approaches; Sec. III is devoted to the presentation of the main results and Sec. IV gives a discussion on the results obtained from the different approaches. Finally, Sec. V describes the main conclusions from the present work.

\section{EXPERIMENTAL AND COMPUTATIONAL DETAILS}

\section{A. Experiment}

The glass samples were prepared by rapid quenching of the room-temperature liquid in a liquid-nitrogen bath.

The inelastic-neutron-scattering experiments were performed using the IN6 time-of-flight spectrometer of the Institut Laue Langevin, Grenoble, France, using an incident wavelength of $4.12 \AA$. The sample container was an aluminum plate of $0.6 \mathrm{~mm}$ internal thickness and the temperature was controlled using a standard orange cryostat. The measurements were performed at temperatures ranging from 5 to $110 \mathrm{~K}$ in the glass phase. At $135 \mathrm{~K}$ a rapid crystallization into the low-temperature $\alpha$ phase occurs, and the measured spectra corresponds to a polycrystal. The spectra at $T=200 \mathrm{~K}$ were taken as an additional reference since at that temperature the melting is complete. Finally calibration runs with the empty cell as well as with vanadium foil of the same thickness as the sample were carried out in order to subtract the container scattering and to normalize the measured intensities. In all cases, the presence (or absence) of crystalization was monitored from plots of the wave-vector dependence of the elastic intensity. The cross-section data were converted into $S(\Theta, \omega)$ dynamic structure factors using the INX (Ref. 10) suite of programs. An absorption correction was applied using a standard $\operatorname{code}^{11}$ and the contribution of multiply scattered neutrons was estimated using a modified version of the DISCUS code. ${ }^{12}$ The constant-angle $S(\Theta, \omega)$ spectra were converted into constant $Q$ by means of the INGRID code. ${ }^{13}$ The vibrational density of states was estimated from the structure factor data using a procedure discussed by Buchenau ${ }^{3}$ following the same lines as detailed in a previous paper. ${ }^{14}$

\section{B. The potential model}

A number of attempts have been reported where model potentials have been used to reproduce thermal and structural properties of the liquid as well as the dynamics of bond breaking and forming of the hydrogen bond (HB) network. ${ }^{15,7,16}$ However, no potential has been validated up to the present moment as able to reproduce the experimentally determined crystal structure or the collective dynamics of this material in the solid or glass phases. On the other hand, due to both the need of performing long MD runs and the fact that the internal molecular modes are, in the condensed phases, ${ }^{17}$ well separated from the lattice ones, a model potential that represents the molecular unit as rigid has been adopted. ${ }^{15}$ Such a potential function, which is of a site-site type, has been shown to reproduce the experimental $g(r)$ partial pair correlation functions in the liquid phase as well as some thermodynamical properties such as the specific heat and the self-diffusion coefficient, ${ }^{15,7}$ and the most salient features of the collective dynamics of the cold liquid. ${ }^{7}$

In order to test the model employed for the interparticle potential, a minimization process using the WMIN code ${ }^{18}$ has been carried out starting with the experimentally determined crystal structure for the lowtemperature $\alpha$ phase, ${ }^{19}$ using the cell parameters, molecular rotation, and translational degrees of freedom as free parameters. The Ewald method ${ }^{20}$ was used to deal with the electrostatic long-range interactions and a cutoff distance of $12 \AA$ has been taken for the Lennard-Jones interactions.

The changes undergone in the process by the three lattice parameters were in percentage units of $2.9,3.8$, and 3.8 , whereas the maximum shift in the atomic coordinates is $0.3 \AA$. Such a discrepancy between calculated and experimental crystal structures indicated that the potential model considered here satisfactorily reproduces the observed structure.

\section{Molecular dynamics}

The trajectory of a 256 methanol molecules system subject to cubic periodic boundary conditions was computed using Newtonian classical mechanics (NVE-P ensemble) for two different thermodynamic states (see Table I). The molecules were treated as rigid bodies composed of six mass points (modeling the atoms in the molecule) and three interaction sites located at the oxygen, carbon, and hydroxylic hydrogen positions. The Cartesian equations of motion were integrated using the velocity version of the Verlet algorithm ${ }^{21}$ with a time step of $10^{-14} \mathrm{~s}$, and the RATTLE algorithm ${ }^{22,21}$ was used to implement the holonomic constraints required to keep all intramolecular distances fixed. The molecular geometry and parameters defining the interaction potential were those proposed by Haughney, Ferrario, and McDonald. ${ }^{15}$

A switch function ${ }^{23}$ was used to turn off smoothly all the interactions between pairs of molecules whose centerof-mass separation was greater than a cutoff distance. 
TABLE I. Average values of simple thermodynamic properties for the simulated glass.

\begin{tabular}{llcc}
\hline \hline Quantity & Units & Run 1 & Run 2 \\
\hline Density & $\mathrm{kg} / \mathrm{m}^{3}$ & 1050.0 & 1055.0 \\
$L_{\text {box }}$ & $\mathrm{nm}$ & 2.4442 & 2.4365 \\
Elapsed time & $\mathrm{ps}$ & 250.0 & 250.0 \\
Temperature $^{\mathrm{a}}$ & $\mathrm{K}$ & $35.205 \pm 0.013$ & $10.202 \pm 0.003$ \\
$U^{\text {inter }}$ & $\mathrm{kJ} / \mathrm{mol}$ & -44.077 & -44.714 \\
Pressure $^{\mathrm{b}}$ & $\mathrm{Atm}$ & 1051.5 & 986.9 \\
$\Omega_{0}^{2}$ & $\mathrm{ps}$ & 533.49 & 550.9 \\
$c_{T}$ & $\mathrm{~m} / \mathrm{s}$ & $2750 \pm 250$ & $2750 \pm 250$ \\
\hline \hline
\end{tabular}

a The standard deviation of the average temperature has been estimated by taking into account the statistical inefficiency of the data (Ref. 21).

b No long-range corrections have been applied.

In addition, long-ranged dipole-dipole interactions were handled by introducing a reaction field with conducting boundary conditions (i.e., $\left.\varepsilon_{\mathrm{RF}}=\infty\right){ }^{24}$ Therefore, the interaction energy between two molecules $\alpha$ and $\beta$ becomes

$U_{\alpha \beta}\left(\mathbf{r}_{\mathbf{i} \alpha}, \mathbf{r}_{\mathbf{j} \beta}\right)=S\left(R_{\alpha \beta}^{2}\right)\left\{U_{\alpha \beta}^{0}\left(\mathbf{r}_{\mathbf{i} \alpha}, \mathbf{r}_{\mathbf{j} \beta}\right)+U_{\alpha \beta}^{\mathrm{RF}}\left(\mathbf{r}_{\mathbf{i} \alpha}, \mathbf{r}_{\mathbf{j} \beta}\right)\right\}$

where $R_{\alpha \beta}$ is the distance between centers of mass for both molecules; and $S(x)$ is the unique fifth-order polynomial satisfying

$$
S(x)= \begin{cases}1 & \text { for } x<R_{L}^{2} \\ 0 & \text { for } x>R_{U}^{2}\end{cases}
$$

and having continuous first and second derivatives at the end points of the interval. ${ }^{23}$ In the present calculations we took $R_{L}=11.70 \AA$ and $R_{U}=12.18 \AA$ for both runs. The function $U_{\alpha \beta}^{0}\left(\mathbf{r}_{\mathbf{i} \alpha}, \mathbf{r}_{\mathbf{j} \beta}\right)$ is the potential function referred to as model H1 by Haughney, Farrario, and McDonald $^{15}$ and has the form of a sum of site-site interactions. Whilst $U_{\alpha \beta}^{\mathrm{RF}}\left(\mathbf{r}_{\mathbf{i} \alpha}, \mathbf{r}_{\mathbf{j} \boldsymbol{\beta}}\right)$ is the term due to the reaction field, which can be expressed as a sum of chargecharge interactions. ${ }^{25}$

A computer-generated glass at $35 \mathrm{~K}$ was produced by means of a temperature quench from an equilibrated liquid run at $300 \mathrm{~K}$. A subsequent quench of an equilibrated configuration corresponding to the equilibrated $35 \mathrm{~K}$ glass yielded the glass at $10 \mathrm{~K}$. The quenches were achieved by continuous rescaling of velocities (i.e., every time step during $75 \mathrm{ps}$ ) in order to reach the target temperatures. Then both glasses were allowed to equilibrate for $110 \mathrm{ps}$ of standard NVE simulation.

In order to test the absence of crystallinity, the relaxed configurations where analyzed by means of computation of the static $g(r)$ pair correlation function for the molecular centers of mass. As an illustrative example, Fig. 1 shows a plot of such a quantity as well as the corresponding magnitude calculated from the atomic positions in the crystalline $\alpha$ phase. The short-range static structure partial pair correlation functions $g(r)$ will be compared with the local arrangement in the polycrystal in a subsequent paper.

The density of states (or generalized frequency spectrum) was calculated in the usual way ${ }^{21}$ as a Fourier cosine transform of the atomic velocity autocorrelation functions (VACF's). The reliability of the calculated autocorrelation functions was evaluated by means of procedures described in a previous paper, ${ }^{7}$ and no measurable artifacts due to recurrence effects were found after analyzing the intermediate scattering functions $F(Q, t)$ for times smaller than $12 \mathrm{ps}$. In order to get some estimation of the statistical quality of the computed functions similar procedures to those described in Ref. 7 were followed. Following a decomposition of the atomic velocities into center-of-mass and rotational components $\left[\mathbf{v}_{i \alpha}(t)=\mathbf{V}_{\alpha}(t)+\mathbf{v}_{i \alpha}^{\text {rot }}(t)\right]$, the total atomic VACF's have been decomposed into their constituents, namely: center of mass, rotational, and their cross-correlation functions.

\section{Lattice dynamics}

A lattice-dynamical calculation has been carried out at the energy-minimized crystal configuration using our own computer code. ${ }^{26}$ The dynamical matrix $D(\mathbf{q})$ is set up in terms of rigid-body translations and rotations using the molecular Born-von Karman formalism within the harmonic approximation: ${ }^{27}$

$$
D_{\alpha \beta}^{i i^{\prime}}\left(\mathbf{q} l k k^{\prime}\right)=\left[m_{\alpha}^{i}(k) m_{\beta}^{i^{\prime}}\left(k^{\prime}\right)\right]^{-1 / 2} \sum_{l^{\prime}} \phi_{\alpha \beta}^{i i^{\prime}}\left(l k, l^{\prime} k^{\prime}\right) \exp \left\{i \mathbf{q}\left[\mathbf{x}\left(l^{\prime} k^{\prime}\right)-\mathbf{x}(l k)\right]\right\} .
$$

Here $k$ and $k^{\prime}$ label the different molecules in the unit cell; $i$ and $i^{\prime}$ represent translational $(t)$ or rotational $(r)$ displacements; $\alpha$ and $\beta$ are $x, y$, and $z$ components with respect to molecular principal inertia axes at the center of mass; and $m_{\alpha}^{i}(k)$ is the molecular mass for $i=t$ and the principal inertia moment $I_{\alpha}(k)$ for $i=r$. The force constant tensor is $\phi_{\alpha \beta}^{i i^{\prime}}\left(l k, l^{\prime} k^{\prime}\right)$, whose components are the second derivatives of the lattice energy with respect 


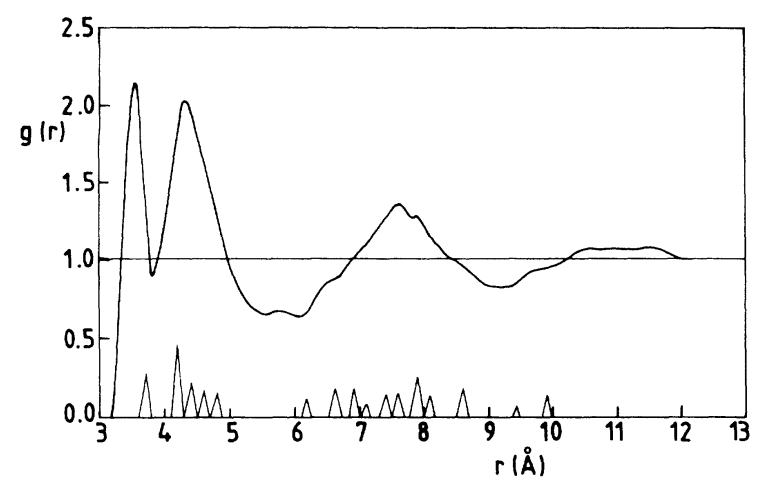

FIG. 1. Center-of-mass $g(r)$ partial pair correlation function computed from the MD simulation at $T=35 \mathrm{~K}$. Triangles denote the set of $\delta$ functions for the polycrystal that have been calculated from an orientational average using the reported crystal structure.

to molecular translations and rotations, whereas $\mathbf{x}(l k)$ is the position vector of the center of mass of molecule $k$ in the unit cell $l$.

The crystal vibrational modes can be obtained from the eigenvalue equation ${ }^{28}$

$$
D(\mathbf{q}) \mathbf{e}(\mathbf{q})=\omega^{2}(\mathbf{q}) \mathbf{e}(\mathbf{q})
$$

where $\omega(\mathbf{q})$ and $e(\mathbf{q})$ are the frequency and polarization vectors normalized to unity of the mode with wave vector q.

The coherent inelastic-neutron-scattering intensity has been obtained in the one-phonon approximation, where the scattering at a dispersion vector $\mathbf{Q}$ is governed by the momentum and energy conservation laws $\mathbf{Q}=\mathbf{G}-\mathbf{q}$ and $E-E_{0}= \pm \hbar \omega(\mathbf{q}) . \quad E$ and $E_{0}$ are the energies of the dispersed and incident neutrons, respectively, $\mathbf{G}$ is a reciprocal lattice vector, and $\omega(\mathbf{q})$ is the frequency of the phonon $\left(\mathbf{q}_{j}\right)$ involved in the process.

The coherent one-phonon scattering function $S(\mathrm{Q}, \omega)$ can be written in the following way for molecular systems: ${ }^{29}$

$S(\mathbf{Q}, \omega)\left(\operatorname{mode} \mathbf{q}_{j}\right)=\delta \frac{\left[E_{j}(\mathbf{q}) \pm 1 / 2 \hbar \omega_{j}(\mathbf{q})\right]}{\omega_{j}^{2}(\mathbf{q})}\left|F_{1}\left(\mathbf{Q}, \mathbf{q}_{j}\right)\right|^{2}$

with

$$
F_{1}\left(\mathbf{Q}, \mathbf{q}_{j}\right)=\sum_{k} \sum_{i} b_{i} e^{-W_{i}} \mathbf{Q} \cdot\left[\mathbf{e}^{\prime t}\left(\mathbf{q}, k_{j}\right)+\mathbf{e}^{\prime r}\left(\mathbf{q}, k_{j}\right) \wedge \mathbf{x}(k i)\right] \exp [i \mathbf{Q} \mathbf{x}(k i)] \exp [i \mathbf{G} \mathbf{x}(k)]
$$

where $i$ labels the different atoms in the molecule $k, b_{i}$ is the coherent scattering length of atom $i, W_{i}$ is the Debye-Waller factor, $\mathbf{x}(k)$ is the position vector of the center of mass of molecule $k$ and $\mathbf{x}(k i)$ is the position vector of atom $i$ belonging to molecule $k$ with respect to its center of mass. The mass-unweighted polarization vector components are defined as

$$
e_{\alpha}^{\prime 1}\left(\mathbf{q}, k_{j}\right)=\left[m_{\alpha}^{i}(k)\right]^{-1 / 2} e_{\alpha}\left(\mathbf{q}, k_{j}\right) .
$$

For the polycrystal, the relevant scattering function $S(Q, \omega)$ must be obtained as an average over all scattering directions $\mathbf{Q}{ }^{30}$ This process has been carried out by dividing the $Q$ space in a fine mesh $(40 \times 40 \times 40$ points in the first Brillouin zone).

The crystal frequency distribution function [density of states (DOS)] has been calculated by means of a sampling over the crystal Brillouin zone using a mesh of $18 \times 18 \times 18$ points along each reciprocal lattice direction.

\section{RESULTS}

\section{A. Experimental results}

Several representative constant- $Q$ spectra corresponding to glass samples are shown in Figs. 2 and 3. As can be clearly seen upon inspection of the figures, a noticeable finite-frequency response is present in all the spectra although it only appears as an unresolved shoulder in the glass samples at lower momentum transfers.

Also, the inelastic peak that becomes visible at large momentum transfers is located at substantially lower frequencies in the glass samples than in their polycrystalline counterpart. The $Q$ dependence of the peak maxima $\omega_{p}$ is, in both cases, depicted in Fig. 4. The parabolic behavior evidenced in such graph is known to be characteristic, in the case of polycrystals, of recoiling processes as has been long recognized. ${ }^{31}$

The polycrystal data were therefore analyzed in terms of recoil scattering from the lattice under the assumption of asymptotic (large- $Q$ ) behavior where the one-phonon contribution to the dynamical structure factors $S^{1}(Q, \omega)$ is assumed to be composed of a sum of Gaussian contributions, so that the total $S(Q, \omega)$ can be written as

$$
S(Q, \omega)=\exp [-2 W(Q)][n(\omega)+1]\left[S^{1}(Q, \omega)+S^{M}(Q, \omega)\right]
$$

and

$$
S^{1}(Q, \omega)=\sum_{j} \frac{2}{\left(\pi \tau_{j}^{2}\right)} \exp \left[-\left(\hbar \omega-\hbar \omega_{0}-\frac{\hbar^{2} Q^{2}}{2 M_{j}}\right)^{2} /\left(2 \tau_{j}^{2}\right)\right]
$$



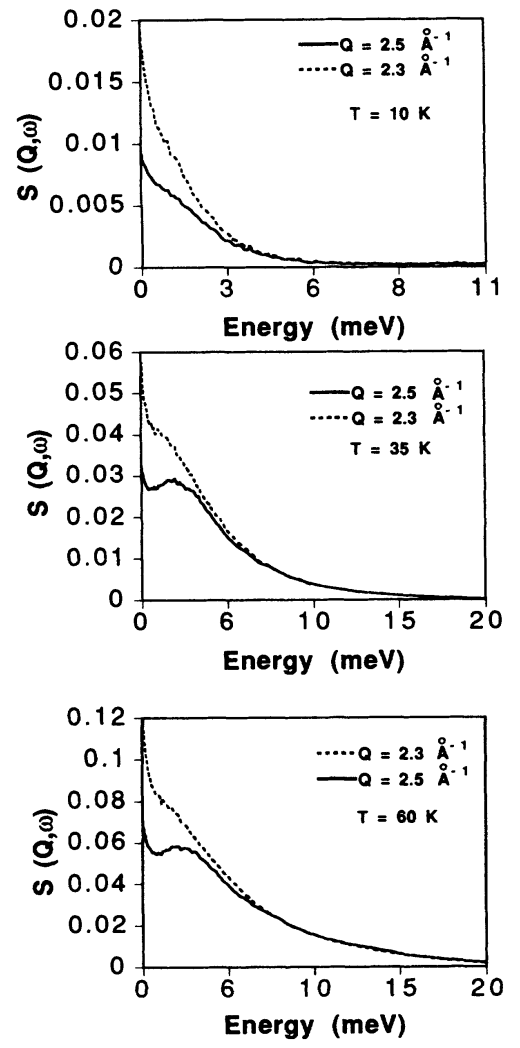

FIG. 2. Constant- $Q$ energy-gain spectra for glass samples at three different temperatures. The curves correspond to interpolations on the constant-angle, $S(\Theta, \omega)$ surface performed using the INGRID code. In order to facilitate the viewing the huge elastic peak has been suppressed.

where the exponential is a Debye-Waller term, $[n(\omega)+1]$ accounts for detailed balancing, and $S^{M}(Q, \omega)$ represents the multiphonon continuum, which is evaluated within the usual convolution approximation. ${ }^{31}$ The sum runs over the number of components into consideration, $M_{j}$ represents the effective mass, $\omega_{0}$ is the recoilless lattice frequency, and the term in $\hbar^{2} Q^{2}$ is a recoil energy. The width factors $\tau_{j}$ behave asymptotically $(Q \rightarrow \infty)$ as

$$
\tau_{j}^{2}=\frac{\hbar Q^{2}}{2 M_{\mathrm{av}}} \int Z(\omega) n(\omega) \omega d \omega
$$

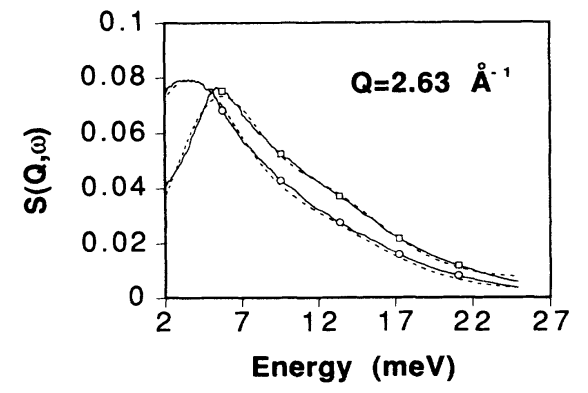

FIG. 3. Energy-gain spectra for polycrystal (solid line with squares) at $T=135 \mathrm{~K}$ and glass (solid line with circles) at $T=110 \mathrm{~K}$. The dashed lines show the fitted function using Eq. (8) (see text).

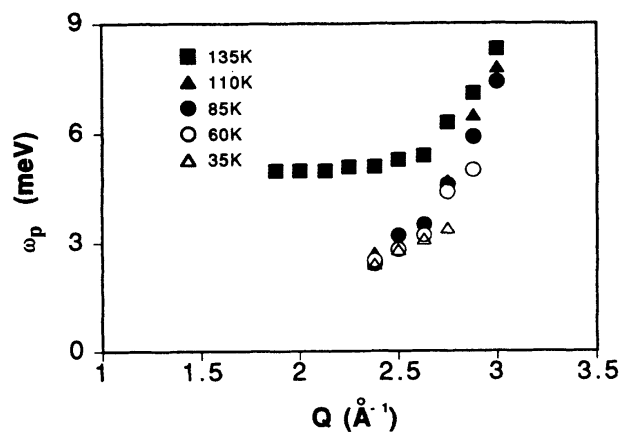

FIG. 4. Wave-vector dependence of the position of the low-frequency peak $\omega_{p}$. The polycrystalline data are given for $T=135 \mathrm{~K}$, and the glass data are shown for $T=110 \mathrm{~K}$, $85 \mathrm{~K}, 60 \mathrm{~K}$, and $35 \mathrm{~K}$, respectively.

Such an approximation represents the dynamic structure factor in terms of Gaussians centered at an energy that is the sum of the lattice and the recoil term with a width that only becomes independent of the momentum transfer in the free-particle limit. Since the achieved range of momentum transfers explored in this work falls short of such a limit, the widths of the individual Gaussians were taken as adjustable parameters. As a matter of fact, several trials using Eq. (10) to estimate the width did not provide acceptable fits to the experimental intensities.

Excellent results were obtained using this approximation, but leaving the widths as adjustable parameters, and some results are shown in Fig. 3. The spectra covering an energy-transfer range of about $25 \mathrm{meV}$ from the elastic line can be described as a sum of two Gaussian distributions centered at frequencies that correspond to the lattice mode plus a recoil contribution. In the polycrystal such lattice frequencies are of about 5.0 and 12.0 $\mathrm{meV}$. The wave-vector dependence of the peak maxima can therefore be accounted for in terms of recoiling motions of the atoms taking part in low-frequency lattice modes, as will be commented on in detail below.

A recoil mass of $1.36 \mathrm{amu}$ (atomic mass units) was computed from the fits, which is in excellent agreement with the Sachs-Teller mass ${ }^{32}$ for the hydrogen atoms in the methanol molecule, and is also close to the freeatom mass of $1.35 \mathrm{amu}$ calculated using a cross-section weighted sum of atomic masses.

A similar procedure was employed to analyze the spectra corresponding to the glass phase. The spectra can also be adequately described in terms of sums of Gaussian functions, although, as it is evident from inspection of Figs. 2 and 3, the frequencies corresponding to the lowest-energy peak are now down to about $2 \mathrm{meV}$. A recoil mass of $1.93 \mathrm{amu}$ was also calculated from the recoil curves. It is difficult to interpret such a difference in effective mass between the polycrystal and glass, although on qualitative grounds it may be explained as due to the lower force constants in the glass than in the crystal. As a matter of fact, an estimate using the effective masses and the $\omega_{0}$ frequencies gives a ratio of force constants between the glass and the crystal of about 0.2 .

As can be seen from Fig. 4, the peak position in the 
glass shows a very weak temperature dependence that can, in principle, indicate the harmonic nature of the excitations giving rise to this peak.

In order to investigate the origin of such peaks, the $Z(\omega)$ vibrational density of states was computed from the structure factor data.

Although in the quasiharmonic approximation, the vibrational contribution to the total density of states (DOS) is supposed to be independent of the temperature, we have calculated this quantity for all the measured temperatures in order to explore the extent in frequencies of the anharmonic contributions. For such a purpose, the DOS for each temperature has been computed within the incoherent approximation by means of a formula that relates the observed structure factors with the sought $Z(\omega),{ }^{3}$
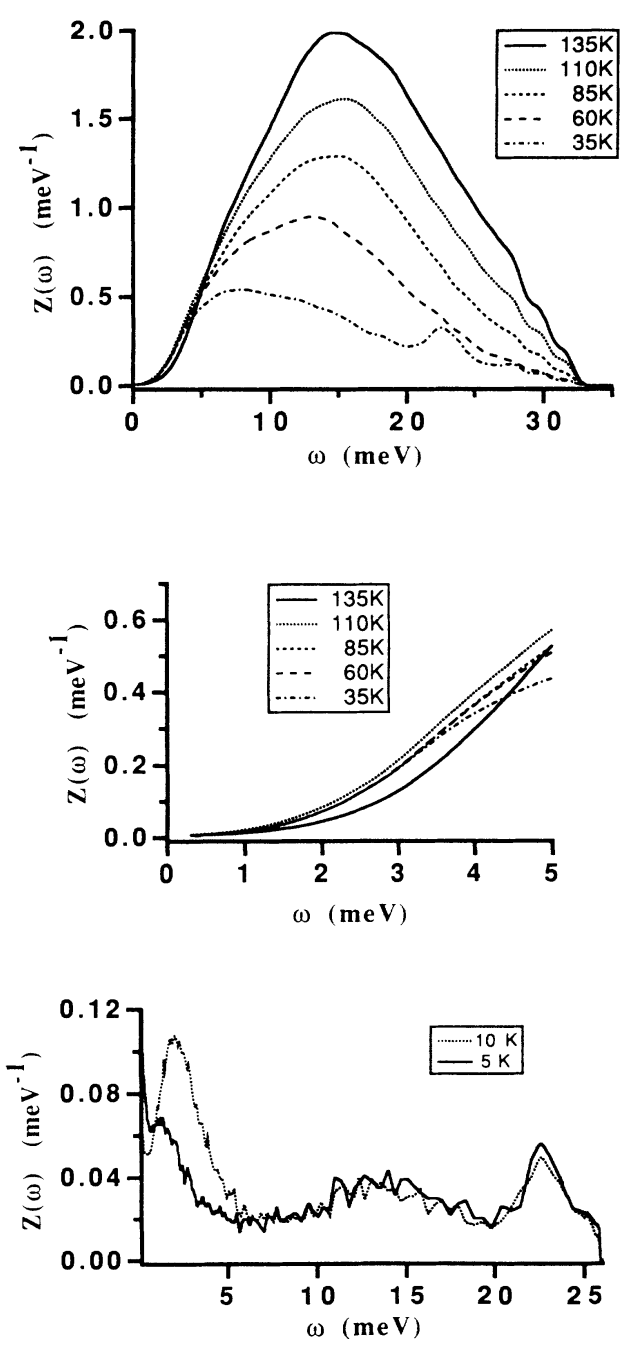

FIG. 5. Vibrational densities of states (DOS) derived from inversion of the experimental spectra (upper box). Comparison of the low-frequency tails of the glass and polycrystalline samples (middle box). Note the enhancement in the DOS corresponding to the glass samples. DOS for the lowest measured temperatures (lower box). The curves have been normalized to $3 k_{B} T$.

$$
S(Q, \omega)=\exp [-2 W(Q)]\left(\hbar Q^{2} / M_{\mathrm{av}}\right)[n(\omega)+1] Z(\omega) / \omega
$$

where the exponential is a Debye-Waller term (or LambMossbauer term), $M_{\mathrm{av}}$ is an average effective mass as calculated from the recoil data, and $[n(\omega)+1]$ is the Bose factor.

A representative sample of the computed distributions $^{33}$ is shown in Fig. 5, where it can be seen that well resolved features are clearly apparent for temperatures around $35 \mathrm{~K}$ and below. The fact that $Z(\omega)$ shows such a noticeable temperature dependence was interpreted as caused by the presence of anharmonic contributions, that become more important as the temperature is raised. As a matter of fact, the importance of other mechanisms that could contribute to this temperaturedependent behavior such as structural changes in the glass with temperature or physical aging were disregarded since only small variations in the glass structure with temperature were found in a neutron-diffraction study, ${ }^{34}$ and the fact that the stability of the glass (a few days) is far longer than the time taken for the measurements.

A comparison between the polycrystal and glass DOS showing in the latter case a noticeable enhancement at low frequencies is shown in the lower part of the figure where it can be seen that for energy transfers below 4 $\mathrm{meV}$ additional excitations are readily apparent in the glass phase.

In order to examine the extent in energy transfers of anharmonic contributions, the behavior of the inelastic intensities at several values of the energy transfer versus temperature was analyzed. Some representative sample plots are given in Fig. 6, where it can be clearly seen that the excitations about $2 \mathrm{meV}$ [i.e., the maxima of $S(Q, \omega)$ in the glass] behave harmonically, whereas such characteristic is lost for higher energy transfers. As can be seen from the plots shown in the figure, the intensity versus temperature curves for energy transfers above $2 \mathrm{meV}$ deviate from linearity, showing a sigmoid behavior that is usually interpreted in terms of the onset of anharmonic motions. The deviation from linearity becomes more important at higher energy transfers as is also evidenced in the figure. Further discussions about this topic are deferred until following sections where a comparison with calculated results will be made.

The $Q$ dependence of the intensity of the elastic peak has been analyzed in order to get an estimate of the $r_{s}$ vibrational mean-squared amplitudes as well as their temperature dependence. Such magnitudes, which are defined through the Lamb-Mossbauer term for recoilless emission $f_{s}=\exp \left(-r_{s} Q\right)^{2}$, can be rationalized in terms of kinetic-theory approaches ${ }^{35}$ as being characteristic "localization lengths," thus giving direct information about the spatial extent of the atomic motions. A plot of the temperature dependence of such magnitudes is shown in Fig. 7, where it can be seen that again the lowesttemperature points show a different behavior than the rest of those corresponding to the glassy regime. As a matter of fact, and contrary to what has been reported 

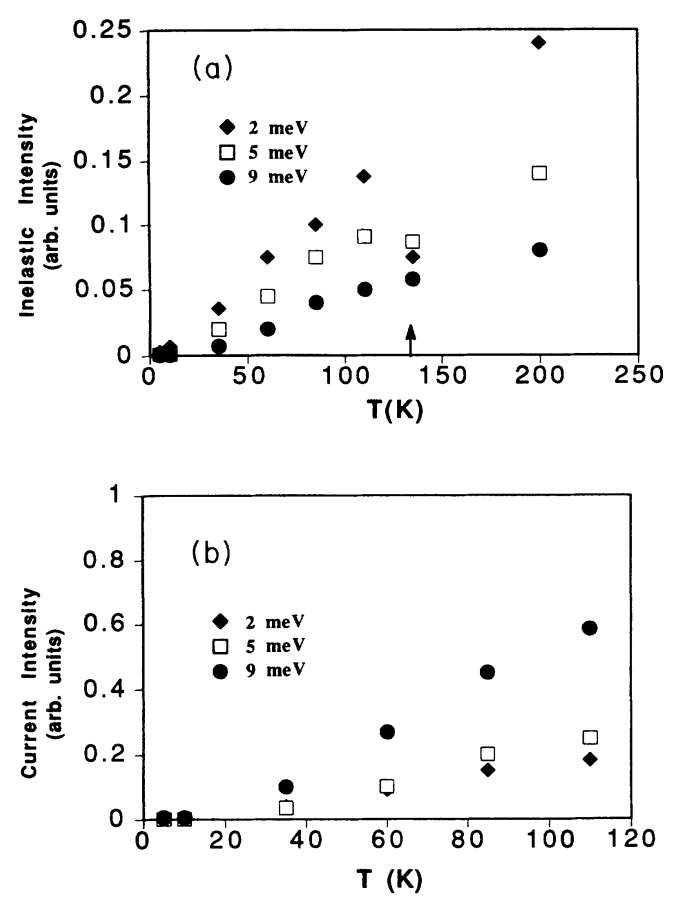

FIG. 6. (a) Temperature dependence of the inelastic intensities at several values of the momentum transfer [i.e., $S(Q, \omega)$ at $\omega=$ const]. The arrow indicates values measured for the polycrystal and the data at $T=200 \mathrm{~K}$ correspond to the melt (liquid) sample. (b) Same meaning as above but the intensities have been calculated from peaks in the $J_{l}=\omega^{2} S(Q, \omega)$ function.

for van der Waals glasses, ${ }^{35}$ a linear dependence with temperature can only be found in the present set of data at temperatures about $10 \mathrm{~K}$ and below. A noticeable departure from linearity of $r_{s}^{2}$ well below the thermodynamical glass transition temperature is therefore clearly apparent, which again contrasts with other organic glasses where such an increase is only visible at temperatures close to $T_{g}$. Furthermore, the value corresponding to the polycrystal at $135 \mathrm{~K}$ lies noticeably below the curve joining the glass and liquid points, which is another confirmation of the softening of force constants in the glass with respect to the crystalline phase.

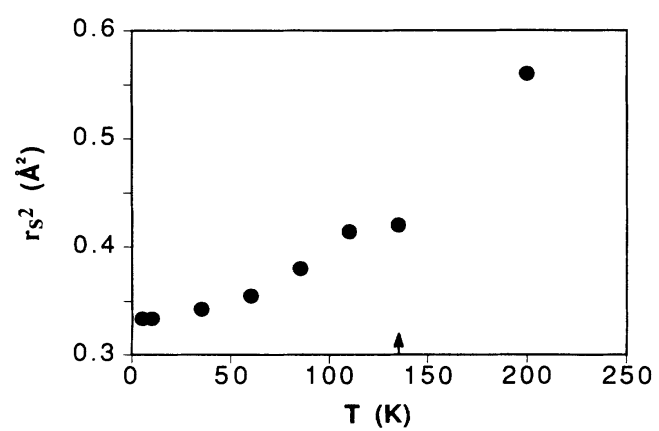

FIG. 7. Temperature dependence of the $r_{s}$ amplitude terms of the Lamb-Mossbauer factors derived from the wavevector dependence of the elastic peak intensities. The arrow marks the data corresponding to the polycrystalline sample.

\section{B. Molecular- and lattice-dynamics results}

A comparison between the DOS obtained from both glass and polycrystal phases calculated from the analysis of MD trajectories and from LD computations is shown in Fig. 8. It can be seen that the frequency distribution function for the crystalline phase of methanol shows very sharp peaks at certain frequencies. Several broad peaks with frequencies in the same range can also be identified in the frequency distribution function for the glassy state. This fact suggests that the crystal modes that contribute to these peaks also survive in some way in the glass phase.

In order to explore this hypothesis, we have identified the nature of the modes belonging to each peak in the crystal phase. The results show that these sharp peaks arise from modes that show very little dispersion, giving rise to flat dispersion hypersurfaces $\omega=\omega(\mathbf{q})$, which are then projected onto a single peak in the frequency distribution function.

It is remarkable that the molecular polarization vectors for the different modes belonging to each peak are rather similar, except for the $32.5-\mathrm{meV}$ peak where polarization vectors of two different kinds can be found. This property, together with the flat character of the $\omega(\mathbf{q})$ dispersion relation indicates that the crystal behaves for these particular modes as an independent Einstein oscillator, in which each molecule vibrates in a potential well produced by the neighboring ones and the movements of molecules in different unit cells are decoupled. This can explain why these modes survive in the glassy state given that they depend mainly on the short-range molecular environment, which is very similar for the crystal
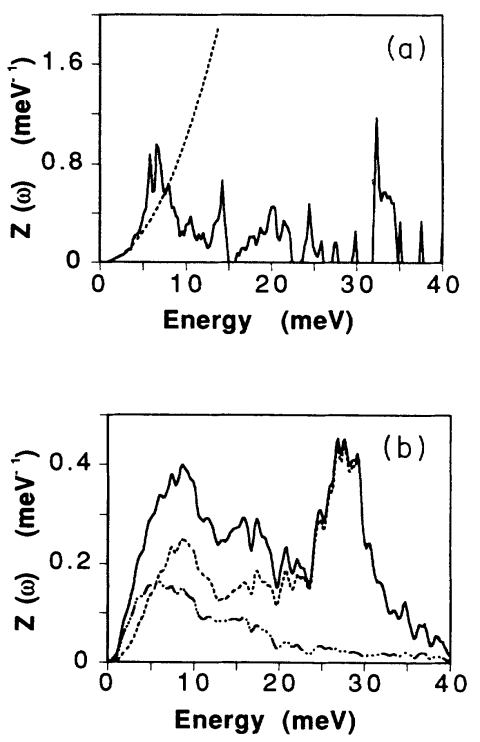

FIG. 8. Vibrational densities of states calculated by means of (a) lattice dynamics, the dashed line is the estimated Debye contribution, and (b) computer molecular dynamics (MD). Note the decomposition into total (solid line), rotational (dashes), and center-of-mass (dash-dot) terms. The curves are normalized so that the total number of states equals $3 k_{B} T$. The calculation was performed at a temperature $T=35 \mathrm{~K}$. 
TABLE II. Normalized averages of the rotational and translational components of the molecular polarization vectors over all the crystal modes giving rise to every peak. Two motions appear at $32.5 \mathrm{meV}$.

\begin{tabular}{lcccccc}
\hline \hline$\hbar \omega(\mathrm{meV})$ & $T_{x}$ & $T_{y}$ & $T_{z}$ & $R_{x}$ & $R_{y}$ & $R_{z}$ \\
\hline 6.7 & 0.200 & 0.228 & 0.356 & 0.742 & 0.468 & 0.105 \\
14.4 & 0.212 & 0.223 & 0.670 & 0.238 & 0.617 & 0.140 \\
20.4 & 0.448 & 0.529 & 0.143 & 0.074 & 0.132 & 0.690 \\
24.6 & 0.877 & 0.124 & 0.082 & 0.137 & 0.049 & 0.433 \\
$32.5(\mathrm{I})$ & 0.282 & 0.515 & 0.083 & 0.276 & 0.117 & 0.747 \\
$32.5(\mathrm{II})$ & 0.022 & 0.016 & 0.187 & 0.920 & 0.344 & 0.018 \\
\hline \hline
\end{tabular}

and the glassy phases.

Table II shows the normalized average of the rotational and translational components of the molecular polarization vectors over all the crystal modes belonging to every peak (two averages for the $32.5-\mathrm{meV}$ peak). They are expressed in the molecular principal-inertia axes $(x$ is the smallest and $z$ the largest inertia axes). As can be judged from the vector components given in the table, all the peaks are originated by modes of mixed (translational and rotational) character. The peak at $6.7 \mathrm{meV}$ shows a predominately rotational character whereas a sharp translational component is manifest at $14.4 \mathrm{meV}$, a frequency that is near the Debye cutoff frequency $\omega_{D}=$ $13.95 \mathrm{meV}$, estimated from the fit of a Debye $Z(\omega)$ to the low-frequency (up to $5 \mathrm{meV}$ ) part of the spectrum. Also a large translational component is readily apparent in the $T_{x}$ contribution of the $24.6-\mathrm{meV}$ peak, and finally, the two mode polarizations that correspond to the $32.5-\mathrm{meV}$ intense sharp feature are of a predominately rotational character.

A decomposition in terms of center of mass, rotational motions, and coupling terms has been performed in the case of MD data and the center-of-mass and rotational contributions are also depicted in the figure. The coupling terms were found to be rather small at low frequencies and only show a noticeable contribution centered at about $14 \mathrm{meV}$, which can be disregarded at sufficiently low temperatures.

In order to assign the main features appearing in the DOS calculated for the glass phase, Fig. 9 shows the atomic contributions to this quantity. The following seems clear upon the inspection of the figure:

(i) The mostly center-of-mass contributions follow an $\omega^{2}$ law at low frequencies (up to $1 \mathrm{meV}$ ). From 1 to 3.5 $\mathrm{meV}$ the MD-DOS for all the atoms show an "excess" of vibrational states with respect the Debye behavior, and a crossover to a dependence lower than $\omega^{2}$ takes place at about $3.5 \mathrm{meV}$. Similar features can be seen in the total DOS per atom, although the crossover now takes place at somewhat smaller frequencies $(3 \mathrm{meV})$.

(ii) The three main regions in the total DOS centered at about 7,15 , and $27 \mathrm{meV}$ have very different contributions from the individual atoms in the molecule.

(iii) The intense peak at $27 \mathrm{meV}$ is mostly originated by rotational contributions from the molecular hydrogens.

(iv) The peaks at 7 and $15 \mathrm{meV}$ have contributions of both rotational and center-of-mass character, although the most intense contributions in the curves that are unweighted by the neutron cross sections arise from those atoms nearer to the molecular center of mass (carbon and oxygen).

A comparison between the experimental and calculated DOS for the polycrystal evidences that for energy transfers larger than the one corresponding to the first maximum in the calculated DOS (i.e., $5.6 \mathrm{meV}$ ), the anharmonic contributions become dominant at $135 \mathrm{~K}$. This makes a quantitative comparison difficult since the LD calculation was carried out within the harmonic approximation. However, as will be shown below, the lowfrequency part of the computed $Z(\omega)$ adequately reproduces the position of the low-energy peak evidenced by the experiment. Furthermore, as is shown in the figure, the $Z(\omega)$ estimated from low-temperature data ( 5 and $10 \mathrm{~K}$ ) show a better agreement with the one computed from MD simulations, as can be expected due to the low

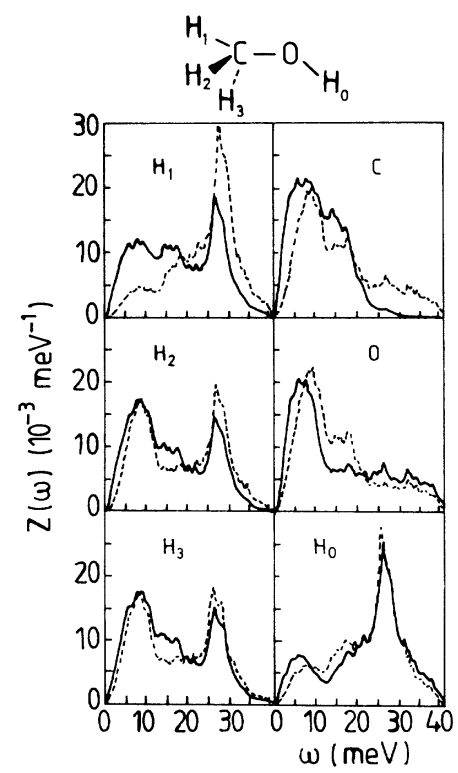

FIG. 9. Total neutron-unweighted densities of states (solid lines) and rotational contributions (dashes) for the different atoms in the molecular unit, as calculated from MD. Atoms $\mathrm{H}_{1,2,3}$ are the methyl group hydrogens, where $\mathrm{H}_{1}$ is in a staggered position with respect to the hydroxyl $\mathrm{HO}$, as is shown in the chemical formula at the top of the graph. 
weight, at these temperatures, of the anharmonic contributions.

In order to gain some insight into the microscopic origin of the modes giving rise to the main peaks in the DOS obtained from the LD calculation the inelastic structure factors [i.e., the quantities $S(Q, \omega=$ const)] have been calculated from the structure factors derived from the LD calculation. As is well known, the wave-vector dependence of the inelastic intensity calculated at energy transfers corresponding to peak frequencies can give an indication of the coherence length associated with the excitations giving rise to such a peak. In this respect, it is worth remembering that, at low frequencies, the excitations are expected to show their acoustic nature in such plots as an oscillation that is in phase with the quantity $Q^{2} S(Q)$ static structure factor, whereas excitation of a different nature will show in such plots peaks centered at momentum transfers that define an average coherence length for the modes contributing to the peak in the DOS. Some results regarding the wave-vector dependence of the inelastic intensities are depicted in Fig. 10. The first graph of the figure shows the calculated wavevector dependence for an energy transfer of $0.26 \mathrm{meV}$. In this case, the two peaks appearing at 1.5 and $1.9 \AA^{-1}$ are in phase with the static structure function $Q^{2} S(Q)$, thus indicating the purely acoustic nature of the excitations existing at these low frequencies. However, such a phase relationship is lost for frequencies corresponding to the intense peak that appears at $14.4 \mathrm{meV}$ in the computed DOS. Furthermore, well-defined peaks appear at $Q=1.4$ $\AA^{-1}$, which defines a characteristic length of $4.5 \AA$ and $Q=0.7 \AA^{-1}$ corresponding to $8.9 \AA$ in real space. As a consequence, the spatial extent of the excitations other than those of acoustic nature appears to be confined to a region of about $10 \AA$. Such a short-range correlation is also apparent from the Einstein-like character of many of the modes, as will be discussed below.

Although it is rather difficult to draw a picture of the molecular motions involved in the excitation peaks appearing in the DOS, from the analysis of the mode eigenvector components it can be stated that the microscopic origin of most of the peaks involve cooperative rotations along the hydrogen bonds.

Several representative differences between the DOS calculated from MD for the glass and those computed from LD for the polycrystal are readily apparent. First of all, the low-frequency part (below $5 \mathrm{meV}$ ) of the MDDOS substantially deviates from Debye behavior, as evidenced in the lower part of Fig. 8, whereas the crystalline DOS is shown to follow a $\omega^{2}$ law with a Debye temperature $\Theta_{D}=162 \mathrm{~K}$. A clear enhancement is evident in the low-frequency part of the glass DOS. Such a feature has its origin in the center-of-mass (c.m.) contributions to the DOS, as can also be seen from the figure.

It becomes difficult to correlate the peak frequencies of the main features of the DOS in both cases since most of
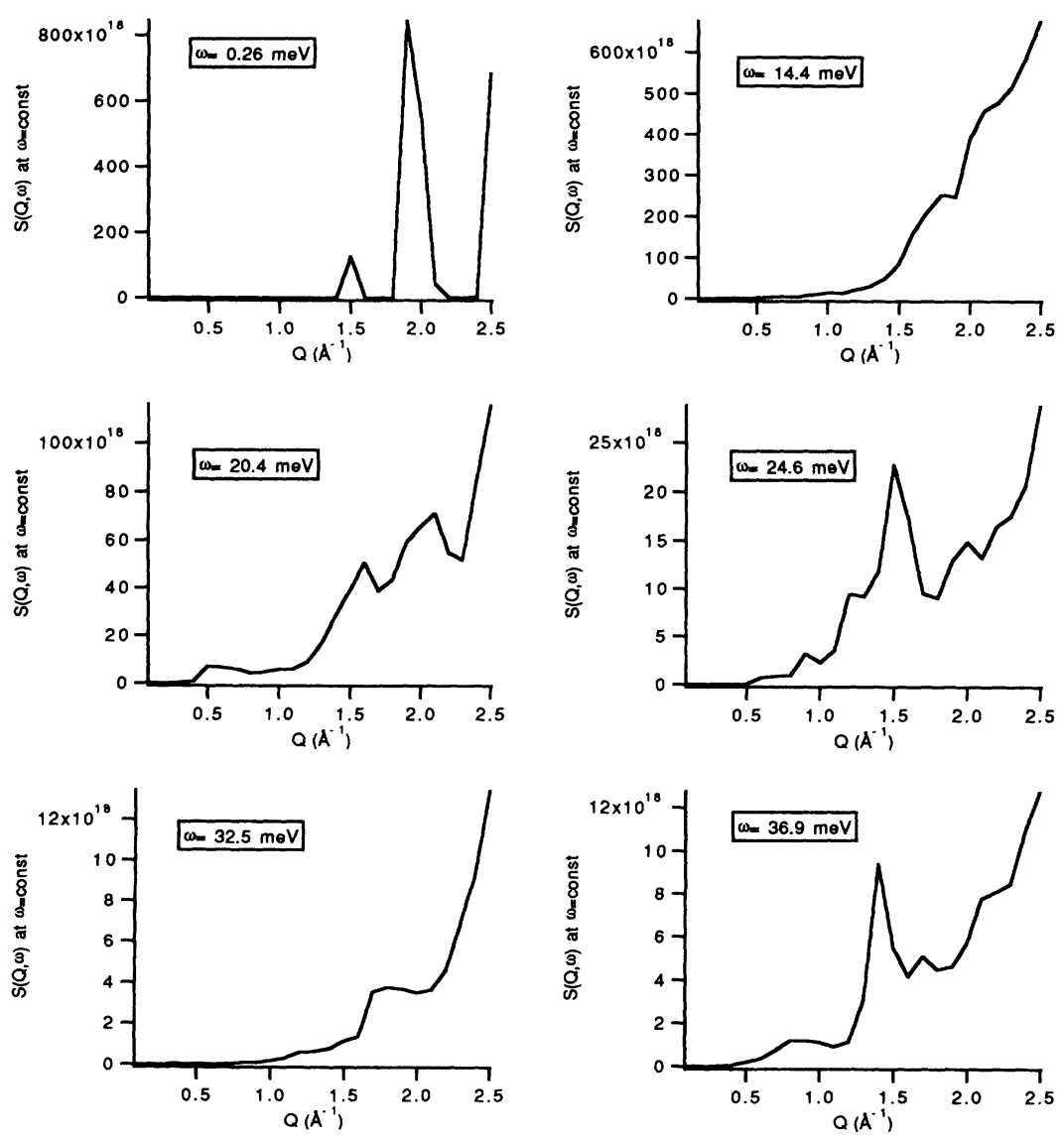

FIG. 10. Inelastic structure factors $S(Q, \omega=$ const) for energy transfers corresponding to the most intense peaks appearing in the DOS. 
the well-resolved features in the polycrystal are blurred in the glass. However, the main rotational peak, which is clearly visible in the glass at about $27 \mathrm{meV}$, can be correlated with the most intense, sharp feature, in the crystal, which is centered at about $32 \mathrm{meV}$, and the same consideration applies to the higher-frequency modes, which in the polycrystal extend up to $50 \mathrm{meV}$ corresponding to isolated optical dispersion branches. Also, the sharp feature located at about $14 \mathrm{meV}$ in the crystal can be related to the broad envelope appearing in the glass at about $12 \mathrm{meV}$, a feature that was evidenced before in the analysis of the experimental spectra in terms of sums of Gaussian functions.

In order to compare the experimental structure factors with those derived from MD simulations and LD calculations, the $S(Q, \omega)$ dynamic structure factors have been computed in both cases from the $Z(\omega)$ functions and corrected by the Bose factors in the incoherent approximation according to Eq. (11). A comparison of the $S(Q, \omega)$ derived from the LD and MD calculations is shown in Fig. 11. Several comments are in order regarding the plots shown in the figure. First of all, the position of the low-frequency, inelastic peak appearing at about $6 \mathrm{meV}$ in the polycrystalline sample is quite well reproduced by the $S(Q, \omega)$ derived from the LD calculation. From inspection of Table II it becomes clear that such a peak corresponds to a combination of lattice modes that correspond to the three low-lying acoustic excitations plus the lowest optical (rotational) components. A detailed discussion of the dispersive behavior of the individual modes will be given in a subsequent paper.
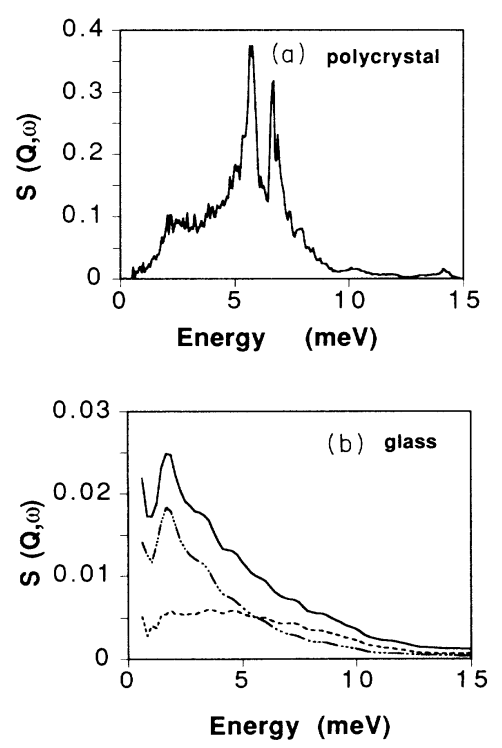

FIG. 11. Calculated $S(Q, \omega)$ dynamic structure factors using Eq. (9) and densities of states for the polycrystal (upper box) and the glass (lower box) for a momentum-transfer $Q=2.5 \AA^{-1}$. The total spectrum is shown by the solid line. The rotational (dashes) and center-of-mass (dash-dots) contributions are also shown. Note that the origin of the lowfrequency peak in the glass is due to the c.m. component of the DOS.
On the other hand, the peak appearing at about $2 \mathrm{meV}$ in the glass samples arises from the low-frequency part of the total DOS, which shows a noticeable enhancement with respect to the one corresponding to the polycrystal, as was shown in Fig. 8. Furthermore, such low-frequency peak arises from the c.m. motions part of the total DOS, as is also illustrated from the figure.

Several other broad features are also visible in both the experimental and calculated spectra, which can be correlated with the different features appearing in the DOS obtained from the LD calculation. In particular, the broad structure centered at about $12 \mathrm{meV}$ and the sharp peak at $27 \mathrm{meV}$ can be assumed to have the same origin as was mentioned above.

\section{DISCUSSION}

The acoustic (mostly translational) character of the low-frequency vibrations that are known to be a universal characteristic of the glassy state ${ }^{36}$ has been evidenced from the concurrent use of INS and computer MD simulation as well as LD in the harmonic approximation for the polycrystalline case. The harmonic character of such modes has been established from the analysis of the temperature dependence of the inelastic intensities. On the other hand, a comparison of higher-frequency vibrations in both crystalline and glass phases shows that, at least for modes giving rise to well resolved peaks (i.e., the one at $32.5 \mathrm{meV}$ in the crystal and those above it), a noticeable softening takes place in the glass phase. Such phenomenon can be explained in terms of the onset of highly anharmonic motions in the glass so that the average frequency $\omega_{\mathrm{av}}$ is reduced by an amount $\Delta \omega$ from the crystal $\omega_{0}$ reference value. From the frequency shifts, it is therefore possible to estimate the order of magnitude of the cubic term of the expansion of the intermolecular potential in small displacements (neglecting quartic and higher-order interactions) $\alpha_{3}$ from ${ }^{37}$

$$
\Delta \omega=-\frac{5 \alpha_{3}^{2}}{12 \omega_{0}^{3}} r_{\text {harm }}^{2}
$$

where the $r_{\text {harm }}^{2}$ term is a mean displacement corresponding to the low-temperature regime where only harmonic motions are to be considered. For such a purpose the $r_{\text {harm }}^{2}$ value was taken from a low-temperature $(15-\mathrm{K})$ diffraction study of the solid $\alpha$ phase $^{19}$ where a value of $0.031 \AA^{2}$ was found. Using this estimate and the $5.5-\mathrm{meV}$ decrease in frequency of the rotational peak, a value for $\alpha_{3}$ of $8.77 \times 10^{27} \mathrm{rad}^{2} \mathrm{~s}^{-2} \AA^{-1}$ is obtained. Such a value contrasts with the one for the quadratic term, which is $\omega_{0}^{2}=2.43 \times 10^{27} \mathrm{rad}^{2} \mathrm{~s}^{-2}$, which is calculated from the frequency of the rotational peak in the polycrystal. The anomalously large value of the cubic term precludes the use of any perturbational approach for any significant calculation of properties such as mean-squared amplitudes of vibration.

A similar characteristic was also noted by Syrykh, Zhernov, and Chernoplekov ${ }^{37}$ in a study of the DOS of amorphous metallic alloys, where the presence of soft lo- 
cal atomic configurations leading to large effects noticeable in the Debye-Waller term was assumed.

Although there have been reported some mechanisms to account for such a kind of frequency reduction where local strains ${ }^{5}$ or some sort of instabilities ${ }^{38}$ are assumed to be the origin of such phenomenon, it has been difficult to apply the proposed schemes for a realistic case such as the one considered in the present work. A tentative scheme to explain the sound-mode softening by localized motions following some ideas recently applied for the study of orientational glasses ${ }^{41}$ will be presented in a subsequent paper.

An attempt to study the temperature dependence of the specific heat at constant volume, $C_{v}$, calculated using the DOS derived from experimental means as well as from MD or LD has also been carried out. The $C_{v}$ curves calculated within the harmonic approximation according to

$$
C_{v}=3 N k \int_{0}^{\omega_{M}}\left(\frac{\hbar \omega}{k_{B} T}\right)^{2} \exp \left(\frac{\hbar \omega}{k_{B} T}\right) /\left[\exp \left(\frac{\hbar \omega}{k_{B} T}\right)-1\right]^{2} Z(\omega) d \omega
$$

where $Z(\omega)$ has been obtained from the computer calculations and from the INS experiments, are shown in Fig. $12(\mathrm{a}),{ }^{39}$ as well as some experimental results of the $C_{p}$ constant-pressure specific heats measured by means of adiabatic calorimetry. ${ }^{40}$

As can be readily seen upon inspection of Fig. 12(a),
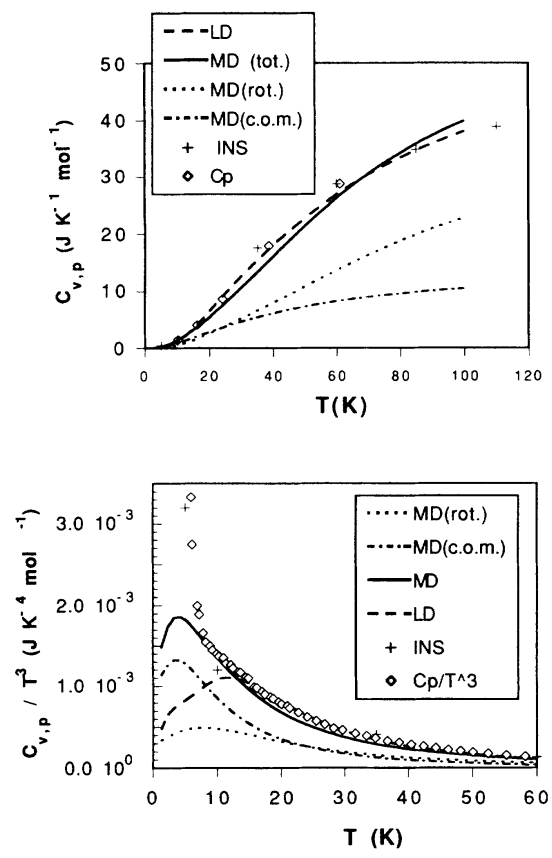

FIG. 12. The upper part shows a comparison of the temperature dependence of the specific heat at constant volume $C_{v}$ calculated from the LD and MD densities of states with the ones obtained from the present set of neutron and calorimetric (for $C_{p}$ ) experiments. The calculations carried out within the harmonic approximation for the computer calculations are shown as MD (total $C_{v}$, i.e., the sum of center-ofmass, rotational, and coupling-terms contribution), as well as the mostly center-of-mass $\mathrm{MD}$ (c.m.) and rotational $\mathrm{MD}$ (rot.) components. The polycrystal data are given as LD and the + markers show the result using the DOS obtained from inversion of the experimental data. The calorimetric data for the glass are shown by open triangles. The lower part of the graph shows the low-temperature region of the $C_{v} / T^{3}$ curves. The same symbols as those in (a) are used. the specific heat calculated from MD substantially differs from the one corresponding to the crystalline, reference state. At temperatures below $5 \mathrm{~K}$ the crystal shows an approximate $T^{3}$ behavior, with a temperature coefficient of $1 \times 10^{-3} \mathrm{~J} \mathrm{~K}^{-4} \mathrm{~mol}^{-1}$ although a clear departure from pure Debye behavior is seen below $1 \mathrm{~K}$, as will be commented on below. The glass data shows a stronger dependence with temperature, which gives a corresponding coefficient in $T^{3}$ of $3.71 \times 10^{-3} \mathrm{~J} \mathrm{~K}^{-4} \mathrm{~mol}^{-1}$ and a more severe departure from the cubic dependence law. Although the $C_{v}$ data can be, up to $T=5 \mathrm{~K}$, approximately accounted for with additional terms with linear and quadratic temperature dependences, we were unable to get a reasonable approximation for the shape of the $C_{v} T^{-3}$ curves using physically meaningful (positive) values for the temperature coefficients.

The fact that all the experimental data lie above the curve corresponding to MD, especially as the temperature is raised, can be interpreted for temperatures above $10 \mathrm{~K}$ as a clear indication of the importance of both anharmonic effects, which were evidenced by comparison of the high-temperature DOS with those calculated from $\mathrm{LD}$ and $\mathrm{MD}$, as well as the contribution of the internal molecular degrees of freedom, which has been neglected in both LD and MD calculations. Such higher-order (anharmonic) processes seem to be equally important in the crystal phase, as can also be gauged from comparison of the polycrystal $Z(\omega)$ with the one calculated by scaling the LD result to the same temperature as shown in Figs. 5 and 8 . However, the strong discrepancy between the calculated and experimental $C_{v}$ for $T=5$ and $10 \mathrm{~K}$ seems to indicate that additional effects are present in the real glass but not in the computer-generated one.

In any case it should be taken into account that at low temperatures where the inelastic intensity is very weak, and below $1 \mathrm{meV}$, the neutron data using the energy resolution achieved in the present work, cannot provide an accurate measurement of the $Z(\omega)$ because of the difficulty of separating the contribution from the huge elastic peak from the inelastic response. Therefore, the data measured at $T=5$ and $10 \mathrm{~K}$ should be affected by large errors, it being difficult to estimate the accuracy of these two points.

In order to explore the low-temperature behavior of the specific-heat curves, Fig. 12(b) shows the functions 
$C_{v}(T) / T^{3}$ corresponding to the computer calculations. Several comments are in order regarding the Fig. 12(b): First and foremost, the MD curve shows a noticeable excess in specific heat with respect to the crystal phase, which gives rise to a well-defined maximum located at about $T=4 \mathrm{~K}$. In contrast, only a broad feature is seen in the LD curve centered at about $12 \mathrm{~K}$. The experimental data (not shown in the graph) lay substantially above the curve calculated from the MD simulation. Several causes may explain this discrepancy, such as anharmonic effects at high temperatures and intrinsically quantum features that are dominant at low temperatures. It is obviously difficult to separate the effects due to anharmonic motions from others that are assumed to be characteristic of the low-temperature properties of glasses. However, it should be remarked that the anharmonic contributions should be greatly reduced at these temperatures, and therefore the reason for such an enhancement should be sought as arising from other phenomena that cannot be accounted for using classical mechanics simulations. Furthermore, it has to be taken into account that a contribution linearly dependent with temperature, which is supposed to be dominant below $1 \mathrm{~K}$, has to be added in order to account for tunneling between two-level systems (TLS's) according to the standard version of the TLS phenomenology. ${ }^{1}$ Since there are no low-temperature measurements of the specific heat for this material (the lowest achievable temperature in the reported calorimetric measurements was about $5 \mathrm{~K}$ ) it was not possible to estimate the value for the coefficient of this linear term.

Also remarkable is the fact that the relatively small deviation from a $T^{3}$ law commented on in the previous section for the polycrystal can be assigned to the rotational contributions to the DOS since an analogous behavior is seen in the figure for the mostly rotational part of the MD-DOS. As can be seen from Fig. 12(b), a clear decrease in specific heat occurs from $2 \mathrm{~K}$ downwards, and at about $0.5 \mathrm{~K}$ the rotational motions are nearly completely frozen.

An attempt to analyze the shape of the pronounced bump at about $4 \mathrm{~K}$ in terms of a sum of temperaturedependent terms with integer or fractional exponents failed to give any reasonable approximation if the coefficients were constrained to be positive.

The presence of such a bump is, therefore, accounted for by the mostly center-of-mass part of the MD-DOS that is peaked at low frequencies (about $6 \mathrm{meV}$ ). Moreover, the shape of such a maximum can be reproduced by calculation of the $C_{v} T^{-3}$ function using a frequency cutoff of $10 \mathrm{meV}$. The origin of this feature can be assigned to the three different frequency regions, which were apparent in the MD-DOS (i.e., a Debye behavior below 1 $\mathrm{meV}$, an increase higher than $\omega^{2}$ between 1 and $3 \mathrm{meV}$, and a higher-frequency region with a weaker $\omega$ dependence ).

Finally, a comparison of the $C_{v} T^{-3}$ curves obtained from $\mathrm{MD}$ as well as the one computed using the DOS corresponding to $T=10$ and $35 \mathrm{~K}$ is given in Fig. 13 . The reason for choosing the neutron data for $T=35 \mathrm{~K}$

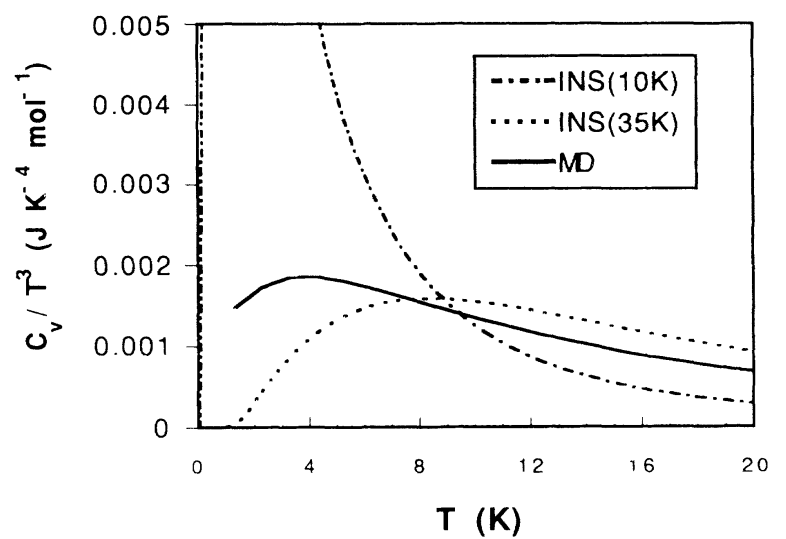

FIG. 13. A comparison between the $C_{v} T^{-3}$ calculated from the simulation (MD) and the one obtained using the DOS corresponding to the measurements at $T=10$ and 35 $\mathrm{K}$.

was, as mentioned above, due to the fact that the measurement at this temperature has better accuracy than the lower-temperature ones. The most noticeable difference between these two curves is the shift in the broad bump from $T=4 \mathrm{~K}$ in the simulation to $T=7 \mathrm{~K}$ in the neutron curve. Such a discrepancy may be readily taken into account from consideration of the fact that, due to the anharmonicity contributions, the DOS at $T=35 \mathrm{~K}$ is peaked at higher frequencies than the one obtained from the simulation. On the other hand, the position in temperature of the maximum calculated using the DOS measured for $T=10 \mathrm{~K}$ is in good agreement with the simulation one, although, as can be seen from Fig. 12(a), the curve had to be scaled to enable the comparison to be made.

\section{CONCLUSIONS}

The present paper constitutes, to the best of our knowledge, the first attempt of analysis of the dynamical response of a molecular glass former in terms of the relevant microscopic motions. In contrast with the situation for glassy metals where the advantages of comparing of computer calculation results ${ }^{42}$ with the measured structure factors were early appreciated, the inherent difficulty in carrying over such calculations for realistic glass-forming molecular systems precluded any discussion in microscopic and quantitative terms. Although some comparisons have been made between experimental results obtained from complex organics and predictions from kinetic theory of the "mode coupling" family, the fact that the predictive power of the kinetic-theory approaches cannot be extended far below $T_{g}$, seems to preclude its applicability to realistic situations where the main focus of interest is in the low-temperature region where clear anomalies are observed.

However, the concurrent use of experiment, MD, and LD has been shown to be a powerful tool for the investigation of the dynamic characteristics of the glass phase, and some of the most relevant conclusions are given below. 
The low-frequency peak in the $S(Q, \omega)$ dynamical structure factor of a molecular glass former has been studied by incoherent INS and simulation means (MD) taking the polycrystal as a reference state. This finitefrequency peak, which is only found in the glass at an energy transfer of about $2 \mathrm{meV}$, is of a mostly translational nature as evidenced from comparison of spectra calculated from the different contributions to the DOS obtained by means of MD simulations. The presence of such a peak is a direct consequence of the presence of "excess" modes in the DOS of the glass, as evidenced by comparison of both experimental and calculated spectra for the glass and polycrystal phases. In opposition, the lowest-frequency peak in the polycrystal at about $6 \mathrm{meV}$ in energy transfer has a rather different nature since the modes giving rise to this finite-frequency response are of mostly rotational origin. Such an "excess DOS" has been evidenced to be of a harmonic nature from the temperature behavior of the inelastic intensities. In this latter respect, it is worth emphasizing the fact that even for this case where the molecular unit shows highly anharmonic internal vibrations the excess DOS still is of purely harmonic nature.

The presence of a noticeable bump in the $C_{v} T^{-3}$ curves is substantiated from a comparison of the results from both MD and experiment as was shown in Fig. 13.

Finally, a comparison with published data for some other glasses such as vitreous silica ${ }^{43}$ or amorphous ice ${ }^{44}$ can be performed in a qualitative basis. From the results presented in this work, it seems clear that for molecular systems the current assignments of the most prominent features appearing in the DOS may be an oversimplification. As a matter of fact, the lowest-frequency peaks appearing in the total DOS in molecular systems in glassy or crystalline states cannot be assigned to excitations of a purely translational character since such kind of excitations (i.e., with no rotational component) is expected to be confined to a frequency range barely reachable by INS. However, MD simulations enable the separation of the mainly translational part contributing to the total DOS. Furthermore, in the particular case of amorphous ice, the substantial crossing of optic branches with the acoustic ones $^{45}$ as well as the presence of low-lying modes whose frequency at the zone boundary falls close to the acoustic ones, will lead to a DOS with characteristics that are common with the one analyzed in the present work.

A similar enhancement in the low-temperature region of the $C_{v} / T^{3}$ curve has been reported for vitreous silica ${ }^{43}$ as well as for some other few glasses, including those of orientational origin ${ }^{2}$, which have been actually measured. ${ }^{46}$ In particular, from the shapes of the curves showing the harmonic contribution to the specific heat obtained from a low-temperature neutron measurement on amorphous ice, ${ }^{44}$ a similar behavior can be inferred. Although such a phenomenon is supposed to be of a universal character, the temperature at which the $C_{v} / T^{3}$ curves show their maxima seems to be strongly sample dependent. It seems therefore natural to call for an extensive intercomparison of the available data in order to see if some scaling law to take into account this dependence can be formulated.

As a final remark a comment regarding some characteristic features found in the DOS of this system as well as some others is in order. It is well known that a crystal phase formation implies collectively coupled movements of molecules far apart. For this reason, we can intuitively expect that a material whose crystalline phase presents a large number of lattice modes behaving according to an Einstein model may have some tendency to form a glassy state, where long-range order is lost. Therefore, we propose as a subject of future research to investigate whether other molecular systems presenting a glassy state also show well-defined sharp peaks in the distribution function of lattice frequencies for the crystalline phase, in order to establish a relation between these two facts.

\section{ACKNOWLEDGMENTS}

The MD calculations were carried out in the ISIS Science Division at RAL, and thanks are given for the allocation of the necessary computational resources. This work has been supported in part by DGICYT Grant No. PB89-0037-C03. The authors greatly acknowledge Dr. R. Burriel from the Instituto de Ciencia de Materiales de Aragon (Zaragoza) for the calorimetric measurements.
*Permanent address: Instituto de Ciencia de Materiales, Dept. Física-Aplicada C-IV, Facultad de Ciencias, Universidad Autónoma de Madrid, E-28049, Spain.

${ }^{1}$ W. A. Phillips, J. Low Temp. Phys. 7, 351 (1972); Rep. Prog. Phys. 50, 1657 (1987); see also S. Hunklinger and A. K. Raychauduri, Prog. Low Temp. Phys. 9, 267 (1986).

${ }^{2}$ U. T. Hochli, K. Knorr, and A. Loidl, Adv. Phys. 39, 405 (1990).

${ }^{3} \mathrm{U}$. Buchenau, in Dynamics of Disordered Materials, edited by D. Richter, A.J. Dianoux, W. Petry, and J. Teixeira (Springer, Berlin, 1989), p. 172.

${ }^{4}$ A. J. Leggett, Physica B 169, 322 (1991); see also C.C. Yu and A. J. Leggett, Comments Condens. Matter Phys. 14, 231 (1988).

${ }^{5}$ T. Egami, K. Maeda, and V. Vitek, Philos. Mag. A 41, 883 (1980).
${ }^{6}$ E. R. Grannan, M. Randeria, and J. P. Sethna, Phys. Rev. Lett. 60, 1402 (1988); Phys. Rev. B 41, 7784 (1990); 41, 7799 (1990).

${ }^{7}$ J. Alonso, F. J. Bermejo, M. García-Hernández, J. L. Martínez, and W. S. Howells, J. Mol. Struct. 250, 147 (1991); see also J. Alonso, F. J. Bermejo, M. GarcíaHernández, J. L. Martínez, A. Criado, and W.S. Howells, J. Chem. Phys. 96, 7696 (1992).

${ }^{8}$ F. J. Bermejo, F. Batallán, J. L. Martínez, M. GarcíaHernández, and E. Enciso, J. Phys.: Condens. Matter 2, 6659 (1990); F. J. Bermejo, F. Batallán, E. Enciso, M. García-Hernández, J. Alonso, and J. L. Martínez, Europhys. Lett. 12, 129 (1990).

${ }^{9} \mathrm{~A}$ preliminary account of Brillouin scattering for the liquid phase has already been given in F. J. Bermejo, R. Ramírez, J. L. Martínez, C. Prieto, F. Batallán, and M. García- 
Hernández, J. Phys.: Condens. Matter 3, 569 (1991); the results concerning the glass phase will be given elsewhere with the coherent scattering results.

${ }^{10}$ Institut Laue Langevin software program INX.

${ }^{11} \mathrm{~J}$. Alonso, M. García-Hernández, F. J. Bermejo, and J. L. Martínez (unpublished).

${ }^{12} \mathrm{M}$. W. Johnson (unpublished).

${ }^{13}$ Computer codes developed by F. Rieutord (Institut Laue Langevin).

${ }^{14}$ F. J. Bermejo, J. L. Martínez, M. García-Hernández, D. Martin, F. J. Mompean, J. Alonso, and W. S. Howells, Europhys. Lett. 15, 509 (1991); some previous results for the coherent response of the deuterated material are given in F. J. Bermejo, D. Martin, J. L. Martínez, F. Batallan, M. García-Hernández, and F. J. Mompean, Phys. Lett. A 150, 201 (1990).

${ }^{15}$ M. Haughney, M. Ferrario, and I. R. McDonald, J. Phys. Chem. 91, 4934 (1987)

${ }^{16}$ P. F. W. Stouten, B. P. van Eijck, and J. Kroon, J. Mol. Struct. 243, 61 (1991).

${ }^{17}$ G. Herzberg, Molecular Spectra and Molecular Structure, II. Infrared and Raman Spectra of Polyatomic Molecules (Van Nostrand Rheinhold, Princeton, NJ, 1945), p. 334. Although vibrational-rotational fine structure can be seen in the high-resolution spectra in the dilute-gas phase, no noticeable bands assignable to internal molecular modes appear in the infrared or Raman spectra of the crystal or liquid below $127 \mathrm{meV}$.

${ }^{18}$ W. R. Busing, Acta Crystallogr. Sec. A 28, S252 (1972).

${ }^{19}$ B. H. Torrie, S. X. Weng, and B. M. Powell, Mol. Phys. 67, 575 (1989).

${ }^{20}$ P. P. Ewald, Ann. Phys. (Leipzig) 64, 253 (1921).

${ }^{21}$ M. P. Allen and D. J. Tildesley, Computer Simulation of Liquids (Clarendon, Oxford, 1987), and references therein.

${ }^{22}$ H. C. Andersen, J. Comput. Phys. 52, 24 (1983).

${ }^{23}$ T. A. Andrea, W. C. Swope, and H. C. Andersen, J. Chem. Phys. 79, 4576 (1983).

${ }^{24}$ M. Neumann, Mol. Phys. 50, 841 (1983).

${ }^{25}$ O. Steinhauser, Mol. Phys. 45, 335 (1982).

${ }^{26}$ A. Criado, A. Conde, and R. Marquez, Acta Crystallogr. Sec. A 40, 696 (1984).

${ }^{27}$ G. S. Pawley, Phys. Status Solidi B 49, 475 (1972).

${ }^{28} \mathrm{M}$. Born and K. Huang, Dynamical Theory of Crystal Lattices (Clarendon, Oxford, 1954).

${ }^{29}$ W. Cochran, Rep. Prog. Phys. 26, 1 (1963).

${ }^{30}$ F. W. Wette and A. Rahman, Phys. Rev. 176, 784 (1968).

${ }^{31}$ M. Warner and J. M. F. Gunn, in Condensed Matter Research Using Neutrons, Vol. 112 of NATO Advanced Study Institute, Series B: Physics, edited by S. W. Lovesey and R. Scherm (Plenum, New York, 1984), p. 289, and references therein.

${ }^{32}$ Calculated from the molecular geometry given in F. J. Bermejo, J. Santoro, F. J. Mompean, D. Steytler, and J. C. Dore, J. Mol. Liq. 33, 187 (1987), by means of the computation of the Sachs-Teller inverse-mass tensor The free- atoms mass is calculated from $M_{\mathrm{free}}=\sum_{j} \sigma_{j} M_{j} / \sum_{j} \sigma_{j}$ where the summations run over all atoms in the molecule, $\sigma_{j}$ is the total cross section, and $M_{j}$ the atomic mass.

${ }^{33}$ Due to the contributions to the intensity arising from the elastic line, data for energy transfers above $0.4 \mathrm{meV}$ were considered only. This problem considerably worsens at the two lowest temperatures, where a substantial overlap is still visible up to $1.2 \mathrm{meV}$. For frequencies above these values and up to $40 \mathrm{meV}$, the derived $Z(\omega)$ should be reliable up to a few percent.

${ }^{34}$ D. C. Steytler, J. C. Dore, and D. C. Montague, J. NonCryst. Solids 74, 303 (1985) .

${ }^{35} \mathrm{~W}$. Gotze in Liquides, Crystallisation et Transition vitreuse, Les Houches Session 51, 1989, edited by J. P. Hansen, D. Levesque, and J. Zinn-Justin (North-Holland, Amsterdam, $1989)$, p. 287.

${ }^{36}$ K. Inoue, T. Kanaya, S. Ikeda, K. Kaji, K. Shibata, M. Misawa, and Y. Kiyanogi, J. Chem. Phys. 95, 5532 (1991).

${ }^{37}$ Equation taken from L. D. Landau and E. M. Lifshitz, Mechanics (Pergamon, Oxford, 1959), Chap. 5. A similar treatment has been applied in G. F. Syrykh, A. P. Zhernov, and N. A. Chernoplekov, Zh. Eksp. Teor. Fiz. 100, 982 (1991) [Sov. Phys. JETP 73, 542 (1991)].

${ }^{38}$ U. Buchenau, Yu M. Galperin, V. L. Gurevitch, and H. R. Schober, Phys. Rev. 43, 5039 (1991).

${ }^{39}$ An extrapolation to $\omega \rightarrow 0$ was used in all cases. For the crystal case a curve on $\omega^{2}$ that joins smoothly the measured DOS up to $2 \mathrm{meV}$ was used. A similar procedure was employed for the glass, using in this case a Debye law with the sound velocity estimated by Brillouin light-scattering, as will be reported in a subsequent paper. The truncations at high $\omega$ were chosen by extending the cutoff until no noticeable variations were apparent in the value of the specific heat. The employed value of the cutoff was $40 \mathrm{meV}$.

${ }^{40}$ Some measurements of $C_{p}=C_{v}+T V \alpha^{2} / \beta+\Delta C_{v}^{\text {anh }}$, where $\alpha$ and $\beta$ are the thermal expansivity and isothermal compressibility, $V$ is the molar volume, and $\Delta C_{v}^{\text {anh }}$ is the anharmonic heat capacity at constant volume, were performed using a Westrum adiabatic calorimeter at temperatures between 5 and $20 \mathrm{~K}$. A full comparison between data from INS, calorimetry, and computer calculations will be reported in due time.

${ }^{41}$ J. P. Sethna, E. R. Grannan, and M. Randeria, Physica B 169, 316 (1991), and references therein.

42 J. Hafner, Phys. Rev. B 27, 678 (1983).

${ }^{43}$ U. Buchenau, M. Prager, N. Nucker, A. J. Dianoux, N. Ahmad, and W. A. Phillips, Phys. Rev. B 34, 5665 (1986).

${ }^{44}$ D. D. Klug, E. Whalley, E. C. Svensson, J. H. Root, and V. F. Sears, Phys. Rev. B 44, 841 (1991).

${ }^{45} \mathrm{H}$. Bilz and W. Kress, Phonon Dispersion Relations in Insulators (Springer, Berlin, 1979), p. 187.

${ }^{46}$ D. G. Cahill and R. O. Pohl, Annu. Rev. Phys. Chem. 39, 93 (1988); see also R. O. Pohl, in Amorphous Solids. Low Temperature Properties, edited by W.A. Phillips (Springer, Berlin, 1981), p. 27. 